\title{
Avaliação de fatores abióticos sobre o período de atividade do graxaim (Cerdocyon thous Carnívora: Canidae)
}

\author{
Fernando Rodrigo Tortato ${ }^{1 *}$ \\ Sérgio Luiz Althoff ${ }^{2}$ \\ 'Panthera, Caixa Postal 3203, CEP 78060-970, Cuiabá - MT, Brasil \\ ${ }^{2}$ Laboratório de Biologia Animal, Universidade Regional de Blumenau - SC, Brasil \\ *Autor para correspondência \\ ftortato@hotmail.com
}

Submetido em 24/03/2009

Aceito para publicação em 25/09/2009

\section{Resumo}

Estudou-se o período de atividade do graxaim (Cerdocyon thous) no vale do Rio Itajaí, Santa Catarina, sul do Brasil através de armadilhas fotográficas durante um período de 15 meses e a relação desta atividade com os fatores abióticos (pluviosidade, temperatura e fases lunares). Foi constatado que sua atividade é basicamente noturna (54\%) e crepuscular (25\%), sendo classificada como catemeral. Não houve nenhuma relação do período de atividade com os fatores abióticos avaliados.

Unitermos: atividade, comportamento, fases lunares, graxaim, pluviosidade, presas, temperatura

\begin{abstract}
Evaluation of abiotic factors on the activity period of crab-eating fox (Cerdocyon thous - Carnivora: Canidae). The activity period of the crab-eating fox (Cerdocyon thous) was studied in the Itajaí valley, Santa Catarina state, southern Brazil, through camera traps during a 15-month survey. The existence of relationships between this behavior and abiotic factors was also investigated. We found that the crab-eating fox's activity is basically nocturnal (54\%) and crepuscular $(25 \%)$. It has been classified as cathemeral. However, there were no relationships among the abiotic factors estimated (rainfall, temperature and lunar phases).
\end{abstract}

Key words: abiotic factors, activity period, behavior, crab-eating fox, prey

\section{Introdução}

O período de atividade é um fator importante para compreender o comportamento de uma espécie (Weller e Bennett, 2001). De acordo com o táxon estudado são verificados diferentes padrões e fatores de influência no período de atividade, sendo que este muitas vezes é considerado uma adaptação às influências ambientais (Beltran e Delibes, 1994).
Pesquisas envolvendo pequenos mamíferos já relacionaram fatores como fases lunares, pluviosidade, umidade relativa do ar com a atividade dos mesmos (Halle e Stenseth, 2000; Everts et al., 2004; Esbérard, 2007) e estudos envolvendo mamíferos de médio porte como Leopardus pardalis (Emmons et al., 1989), Chrysocyon brachyurus (Sábato et al., 2006) e Aotus azarai (Fernandez-Duque, 2004) constataram o efeito de diferentes fases lunares no período de atividade. 
O graxaim (Cerdocyon thous) é um canídeo com ampla distribuição na América do Sul, sendo encontrado desde o Uruguai e norte da Argentina até a Colômbia, ocupando diversos ambientes, sendo que no Brasil ocorre tanto em áreas de floresta como de campo (Berta, 1982; Nowak, 1999) e com exceção da Amazônia é encontrado em todos os biomas (Cerrado, Caatinga, Pantanal, Floresta Atlântica e Campos Sulinos) (Fonseca et al., 1996; Emmons e Feer, 1999; Reis et al., 2006).

O período de atividade do graxaim é predominantemente noturno e crepuscular, mas apresenta eventualmente atividade diurna (Silva, 1994; Cimardi, 1996; Emmons e Feer, 1999; Rodríguez e Nassar, 1999; Maffei e Taber, 2003). No entanto, os fatores que influenciam este comportamento são pouco conhecidos.

O presente estudo buscou avaliar se o período de atividade desta espécie é influenciado por fatores abióticos, como: precipitação, fases lunares e temperatura.

\section{Materiais e Métodos}

\section{Área de estudo}

O estudo foi realizado na Reserva Biológica Estadual do Sassafrás (RBES) e zona de entorno da mesma. A RBES esta inserida na região do médio vale do Rio Itajaí e é dividida em duas glebas, sendo que o presente trabalho foi realizado na Gleba Maior, que possui uma área de 3.682,40ha situada na localidade de Alto Forcação, município de Doutor Pedrinho (S26 $42^{\prime} \mathrm{e}$ W49 $40^{\prime}$ ') e em parte do entorno. O clima é Mesotérmico Úmido sem estação seca (Cfa) com pluviosidade de $1.400 \mathrm{~mm}$ a $1.500 \mathrm{~mm}$ anual. A temperatura média anual é de $19^{\circ} \mathrm{C}$ (GAPLAN, 1986). A área amostrada da RBES fica aproximadamente na cota altimétrica de $850 \mathrm{~m}$.

A RBES se encontra em uma área de transição entre a Floresta Ombrófila Densa e Floresta Ombrófila Mista, tendo como destaque um grande adensamento de canela-sassafrás (Ocotea odorifera) que originou o nome da mesma. Nas décadas de 60 e 70 do século passado houve extração de madeira em toda região, inclusive dentro dos limites da RBES, restando áreas de vegetação secundária caracterizadas por espécies como vassourão-branco (Piptocarpha angustifolia), vassourão-preto (Vernonia discolor), canela-guaica (Ocotea puberula) e a bracatinga (Mimosa scabrella) que representam excelentes indicadores de comunidades primárias alteradas (Klein, 1980; Santos, 2002).

Parte do estudo foi realizada na zona de entorno, particularmente na Fazenda Cerro Azul, caracterizada pela predominância de reflorestamentos de Pinus sp. e áreas em estágio intermediário de regeneração florestal. $\mathrm{O}$ estudo nesta fazenda foi realizado somente em áreas limítrofes a RBES, utilizando-se aceiros e estradas da propriedade.

\section{Procedimento amostral}

Foram utilizadas seis armadilhas fotográficas Tigrinus ${ }^{\circledR}$, compostas por uma câmera fotográfica automática modelo Canon BF10 QD, utilizando filmes fotográficos FUJI ASA 200-400 de 36 poses e pilhas AA alcalinas.

As campanhas foram realizadas de setembro de 2006 a novembro de 2007 com um esforço amostral de 456 dias em 65 pontos distintos, totalizando 2736 armadilhas/dia. Filmes e pilhas foram substituídos a cada 20 dias e as armadilhas remanejadas de local a cada 40 dias. Os locais foram selecionados de acordo com a presença de vestígios de mamíferos, preferencialmente em trilhas existentes. As armadilhas foram instaladas em troncos de árvores a $30 \mathrm{~cm}$ do chão, com auxílio de extensor de borracha. Não se utilizou de iscas para atrair os mamíferos. Foram considerados como registros, aqueles que ocorreram em um intervalo de no mínimo uma hora entre uma foto e outra em um mesmo ponto.

As armadilhas foram dispostas de forma a minimizar possíveis lacunas entre os pontos de amostragem, distribuídas de forma homogênea em uma área previamente determinada de $400 \mathrm{ha}$, com espaçamento mínimo de $200 \mathrm{~m}$ entre as armadilhas. O período de atividade do graxaim foi avaliado a partir do acúmulo de registros em classes de horário, divididos em: período diurno, noturno e crepuscular. Os horários de nascer e por do sol foram definidos através de um cálculo baseado na coordenada geográfica da área estudada e o software Moonrise 3.5 (Sidell, 2002). O 
período crepuscular foi considerado o intervalo de uma hora antes e depois do nascer e por do sol; o período noturno o intervalo de uma hora após o por do sol e uma hora antes do nascer do sol; o período diurno o intervalo entre uma hora após o nascer do sol e uma hora antes do por do sol.

A classificação da atividade da espécie estudada foi baseada em Van Schaik e Griffiths (1996), sendo a espécie considerada diurna quando menos de $10 \%$ dos registros for obtido no período noturno, espécie considerada noturna onde mais de $90 \%$ dos registros for obtido no período noturno e espécie considerada catemeral quando no período noturno forem obtidos entre $10 \%$ e $90 \%$ dos registros.

Verificou-se a existência de diferenças na atividade do graxaim com a pluviosidade e fases lunar utilizado o teste de Qui-quadrado $(\chi 2)$. Para a pluviosidade, primeiramente foi averiguada a possível existência de uma diferença natural entre os dias com e sem chuva no período de estudo, o que poderia direcionar as análises previamente para uma condição.
Para verificar a existência de alguma relação entre a atividade do graxaim em relação à temperatura, utilizouse a Análise de Regressão, relacionado às médias diárias em classes de $1^{\circ} \mathrm{C}$ e a média de registros para cada classe. A utilização da média para cada classe teve a finalidade de retirar o efeito da diferença nas frequências de cada média de temperatura diária, no período amostrado.

\section{Resultados}

Foram obtidos 91 registros fotográficos de graxaim durante o estudo, com $54 \%(\mathrm{n}=49)$ no período noturno, $25 \%(n=23)$ no período crepuscular e $21 \%(n=19)$ no período diurno. O período de atividade do graxaim durante toda a amostragem obteve picos de registros às 04 h e às 20 h (Figura 1).

A pluviosidade de todo o período quando comparando o número de dias com chuva e sem chuva não apresentou diferenças estatisticamente significativas $\left(\chi^{2}=2,53, p>0,05\right)$. Considerando somente os dias onde se obteve registros de graxaim, ocorreram em 39 (43\%) dias com precipitação e 52 (57\%) dias sem precipitação,

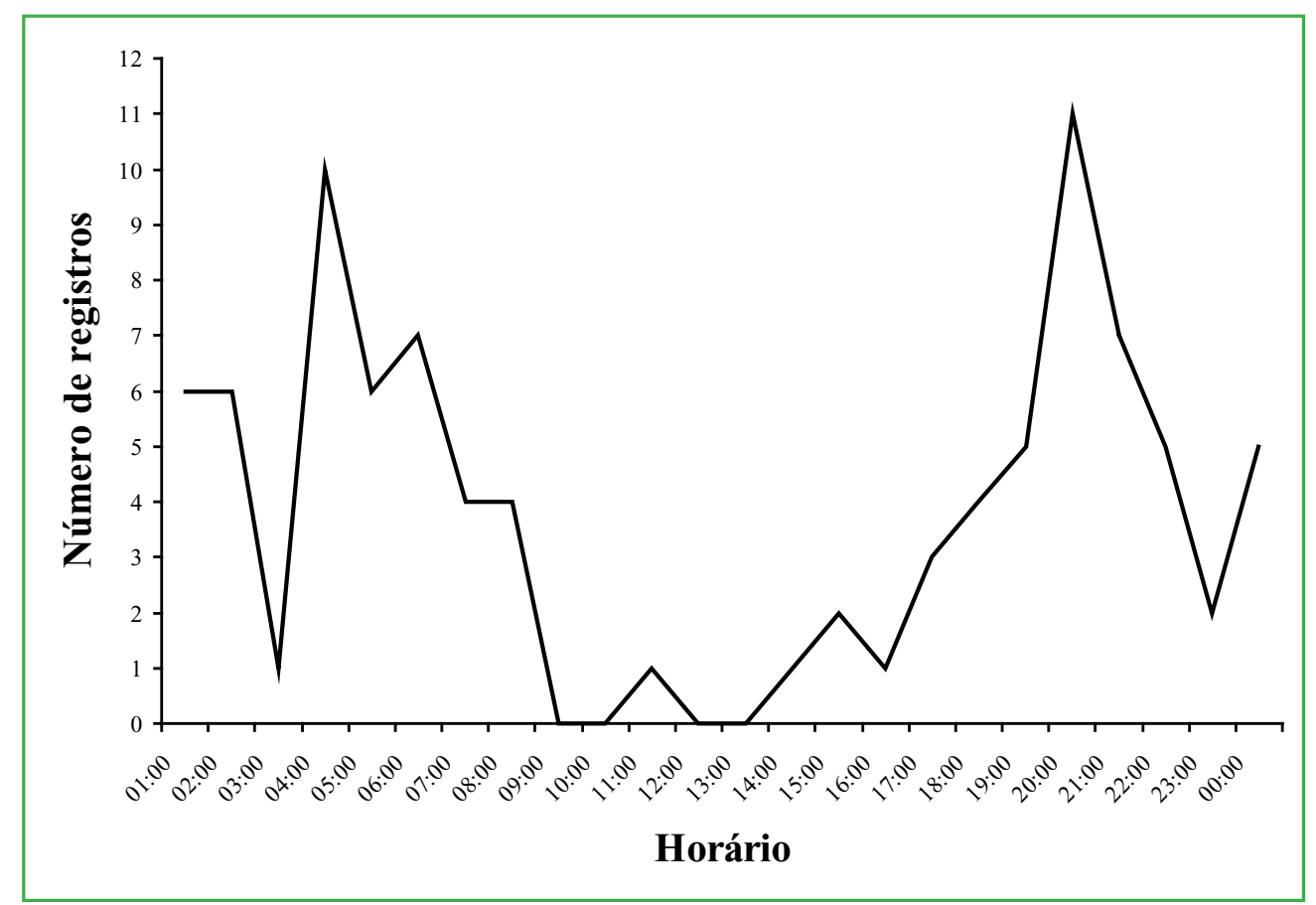

FIGURA 1: Distribuição do período de atividade de Cerdocyon thous na Reserva Biológica Estadual do Sassafrás entre setembro de 2006 e novembro de 2007. 
não havendo diferença estatisticamente significativa na atividade entre os dias com chuva e sem chuva $\left(\chi^{2}=1,85, \mathrm{p}>0,05\right)$.

Para a análise referente a fases lunares, foram considerados primeiramente somente os registros noturnos de graxaim, a comparação através do $\chi^{2}$ não apresentou diferenças estatisticamente significativas $\left(\chi^{2}=3,83 ; p>0,05\right)$.

Incluindo os registros diurnos e crepusculares na análise, com a premissa de que as diferentes fases lunares poderiam influenciar o comportamento diurno desta espécie não foram encontradas diferenças estatísticas $\left(\chi^{2}=4,48 ; \mathrm{p}>0,05\right)$. Na possibilidade de haver alguma fase que difira entre si, foi efetuada a comparação em pares e também se constatou que não houve diferença estatisticamente significativa no período de atividade do graxaim (Tabela 1).

TABELA 1: Comparação entre as fases lunares na atividade de Cerdocyon thous, através do teste Qui-quadrado $\left(\chi^{2}\right)$, realizado na Reserva Biológica Estadual do Sassafrás entre setembro de 2006 e novembro de 2007.

\begin{tabular}{c|c|c|c}
\hline & Minguante & Nova & Crescente \\
\hline Cheia & $\chi^{2}=0,133$ & $\chi^{2}=2,33$ & $\chi^{2}=0,36$ \\
\hline Minguante & & $\chi^{2}=3,52$ & $\chi^{2}=0,92$ \\
\hline Nova & & & $\chi^{2}=0,885$ \\
\hline
\end{tabular}

Em relação à temperatura, foram analisados 89 registros relacionados com 63 dias, onde não se encontrou relação entre a atividade de $C$. thous e a temperatura média diária $\left(\mathrm{R}^{2}=0,0231\right)$.

\section{Discussão}

Neste estudo a atividade do graxaim foi considerada catemeral, não ocorrendo nenhuma relação entre os fatores abióticos e o período de atividade.

Maffei e Taber (2003), monitorando graxains através de radiotelemetria, constataram que a espécie é predominantemente noturna, mas em dois dias de fortes chuvas e temperaturas baixas apresentaram uma maior atividade durante o período diurno, sendo que neste caso a atividade do graxaim foi influenciada por fatores abióticos. Porém em duas situações muito específicas e difíceis de serem detectadas através do uso de armadilhas fotográficas. A atividade do graxaim já foi relacionada à termorregulação (Rodríguez e Nassar, 1999).

Estudos utilizando-se de armadilhas fotográficas não obtêm informações sequenciais do período de atividade, mas sim registros isolados, com isso não sendo possível monitorar constantemente este comportamento como quando realizado em estudos com emprego de radiotelemetria (e.g. Maffei e Taber, 2003). Armadilhas fotográficas são ferramentas cada vez mais utilizadas em campo, principalmente em estudos com mamíferos silvestres, seu uso pode ser potencializado adaptando a este equipamento termômetros e pluviômetros que possam estar registrando a temperatura e pluviosidade no exato local e momento onde ocorrem os registros e assim relacionar com mais clareza a atividade das espécies com estes fatores.

Entre as fases lunares, não ocorreu nenhuma relação com a atividade do graxaim, sendo que as duas fases com maior número de registros, a fase de lua nova e fase de lua cheia são antagônicas em relação à luminosidade. Sábato et al. (2006) constatou para outra espécie de canídeo neotropical (Chrysocyon brachyurus) alterações no período de atividade em diferentes fases lunares. É importante salientar que alguns fatores podem influenciar na luminosidade causada pela lua no ambiente, como a copa das árvores e principalmente dias com céu nublado.

Os mamíferos carnívoros frequentemente têm seu período de atividade influenciado ao período de atividade de suas presas (Lima, 1998; Griffin et al., 2005). Zielinski et al. (1983) estudando a atividade de doninhas (Carnivora-Mustelidae-Martes americana), constatou que a atividade destas, coincidia com a de suas presas, mesmo este período não sendo o melhor em relação a temperatura do ambiente. De acordo com Maffei e Taber (2003), o período de atividade do graxaim coincide com o de suas presas, principalmente os pequenos roedores (Muridae). Neste estudo não podemos relacionar a atividade do graxaim com a atividade de suas presas, pois haveria a necessidade de conhecer sua dieta na área 
estudada e posteriormente avaliar o período de atividade dos vertebrados encontrados.

A estratégia de alimentação deste animal é oportunista, sobrevivendo em áreas degradadas e antrópicas (Langguth, 1975; Motta Junior et al., 1994; Faccure e Monteiro Filho, 1996), com uma dieta onívora incluindo principalmente pequenos mamíferos, aves, insetos e frutas, consumindo a comida mais abundante em cada estação (Bisbal e Ojasti, 1980; Berta, 1987; Motta Junior et al., 1994), tendo estes itens forte ligação com fatores abióticos.

Possivelmente atividade do C. thous é determinada por diversos fatores, estudos mais complexos poderão elucidar quais destes realmente influenciam a atividade desta espécie.

\section{Agradecimentos}

Agradeço a MOBASA - Modo Battistella de Reflorestamento LTDA., Tigrinus Equipamentos para Pesquisa, Fundação do Meio Ambiente de Santa Catarina - FATMA, EPAGRI em nome de Vera Lucia da Silva e Aderbal Domingos Tortato pelo apoio financeiro e logístico; Adrian Eisen Rupp, Gregory Thom e Silva, Jairo Claudino dos Santos, José Correa, Maria Correa, Marcos Adriano Tortato e Carlos Zimmermann pela ajuda e companhia em campo, aos revisores anônimos que colaboraram com sugestões para melhoria deste trabalho e principalmente a Geraldo Moretto e Walfrido Moraes Tomas pelas sugestões e ajuda na análise dos dados.

\section{Referências}

Berta, A. 1982. Cerdocyon thous. Mammalian Species, 186: 1-4. Berta, A 1987. Origin, diversification and zoogeography of the South American Canidae. Fieldiana Zoology, New Series, 39: 455-471.

Beltran, J.; Delibes, M. 1994. Environmental determinants of circadian activity of free-ranging Iberian lynxes. Journal of Mammalogy, 75: 382-393.

Bisbal, F. J. 1986. Food habits of some Neotropical carnivores in Venezuela (Mammalia, Carnivora). Mammalia, 50 (3): 329-339.

Cimardi, A. V. 1996 Mamíferos de Santa Catarina. Fundação de Amparo à Tecnologia e Meio Ambiente, Florianópolis, Brasil, 302 pp.
Emmons, L. H.; Feer, F. 1999. Mamíferos de los bosques húmedos de América Tropical. Ed. FAN, Santa Cruz, Bolívia, 298pp.

Emmons, L. H.; Sherman, P.; Bolster, D.; Goldizen, A.; Terborgh, J. 1989. Ocelot behavior in moonlight. In: Redford, K. H., Eisenberg, J. F. (Eds). Advances in Neotropical Mammalogy. Brill, Leiden, Netherlands, p.233-242.

Esbérard, C. E. L. 2007. Influência do ciclo lunar na captura de morcegos Phyllostomidae. Iheringia, Série Zoologia, 97 (1): 8185.

Everts, L. G.; Strijkstra, A. M.; Hut, R. A.; Hoffmann, I. E.; Millesi, E. 2004. Seasonal variation in daily activity patterns of free-ranging European ground squirrels (Spermophilus citellus). Chronobiology International, 21 (1): 57-71

Facure, K. G.; Monteiro-Filho, E. L. A. 1996. Feeding habits of the crab-eating fox, Cerdocyon thous (Carnivora, Canidae), in a suburban area of southeastern Brazil. Mammalia, 60 (1): 147-149.

Fernandez-Duque, E. 2004. Influences of moonlight, ambient temperature, and food availability on the diurnal and nocturnal activity of owl monkeys (Aotus azarai). Behavioral Ecology and Sociobiology, 54 (5): 431-440.

Fonseca, G. A. B. da; Herrmann, G.; Leite, Y. L. R.; Mittermeier, R. A.; Rylands, A. B.; Patton, J. L. 1996. Lista anotada dos mamíferos do Brasil. Occasional Papers in Conservation Biology, 4: 1-38.

GAPLAN. 1986. Atlas de Santa Catarina. Aerofoto Cruzeiro, Rio de Janeiro, Brasil, 173pp.

Griffin, P. C.; Griffin, S. C.; Waroquiers, C.; Mills, L. S. 2005. Mortality by moonlight: Predation risk and the snowshoe hare. Behavioral Ecology, 16: 938-944.

Halle, S.; Stenseth, N. C. 2000. Activity patterns in small Mammals: An ecological approach. Springer, Berlin, Germany, 320pp.

Langguth, A. 1975. Ecology and evolution in the South American canids. In: Fox, M. W. (Ed.). The wild canids: their systematics, behavioral ecology and evolution. Van Nostrand Reinhold Company, New York, USA, p.192-206.

Lima, S. L. 1998. Nonlethal effects in the ecology of predator-prey interactions. Bioscience, 48: 25-34.

Maffei, L.; Taber, A. B. 2003. Area de acción, actividad y uso de hábitat del zorro patas negras, Cerdocyon thous, en un bosque seco. Mastozoologia Neotropical, 10 (1): 154-160.

Motta Junior, J. C.; Lombardi, J. A.; Talamoni, S. A. 1994. Notes on crab-eating fox Dusicyon thous seed dispersal and food habits in southeastern Brazil. Mammalia, 58: 156-159.

Nowak, R. M. 1999. Walker's mammals of the world. v.1. The Johns Hopkins University Press, Baltimore, USA, 836pp.

Klein, R. M. 1980. Ecologia da flora e vegetação do Vale do Itajaí (Continuação). Sellowia, 32: 164-389.

Reis, N. R.; Peracchi, A. L.; Pedro, W. A.; Lima, I. P. 2006. Mamíferos do Brasil. Editora da UEL, Londrina, Brasil, 437pp.

Rodríguez, G. A. C.; Nassar, J. M. H. 1999. Ecological data on Cerdocyon thous in Barlovento region, state of Miranda, Venezuela. Acta Biologica Venezuelica, 19 (4): 21-26.

Santos, J. S. C. 2002. Questão fundiária da Gleba Maior da Reserva Biológica Estadual do Sassafrás. Monografia de Bacharelado, Universidade Regional de Blumenau, Brasil, 62pp. 
Sábato, M. A. L.; Melo, L. F. B.; Magni, E. M. V.; Young, R. J.; Coelho, C. M. 2006. A note on the effect of the full moon on the activity of wild maned wolves, Chrysocyon brachyurus. Behavioral Processes, 73: 228-230,

Sidell, B. P. 2002. Moonrise 3.5 (32Bit) Software.

Silva, F. 1994. Os mamíferos do Rio Grande do Sul. Fundação Zoobotânica do Rio Grande do Sul, Porto Alegre, Brasil, 246pp.

Van Schaik, C.; Griffiths, M. 1996. Activity periods of Indonesian rain forest mammals. Biotropica, 28: 105-122.

Weller, S. H.; Bennett, C. L. 2001. Twenty-four hour activity budgets and patterns of behavior in captive ocelots (Leopardus pardalis). Applied Animal Behaviour Science, 71 (1): 67-79.

Zielinski, W. J.; Spencer, W. D.; Barrett, R. H. 1983. Relationship between food habitats and activity patterns of pine martens. Journal of Mammalogy, 64 (3): 387-396. 